\title{
Experiência Global no Fechamento Percutâneo do Canal Arterial
}

\section{Carlos Augusto Cardoso Pedra, Simone Rolim Fenandes Fontes Pedra, César Augusto Esteves, Sérgio Luís Navarro Braga, Maria Aparecida Paula Silva, Tarcísio Luís Valle de Almeida, Maria Virgínia Tavares Santana, José Eduardo Moraes Rego Sousa, Valmir Femandes Fontes}

São Paulo, SP

\begin{abstract}
Objetivo - Avaliar a experiência global da nossa instituição com o fechamento percutâneo do canal arterial.

Métodos - Desde dezembro/92, 150 pacientes foram submetidos a 178 procedimentos (85 - técnica de Rashkind; 87 - coils de Gianturco; 6 - coils Duct Occlud). A mediana de idade foi de 6,5 anos (1 a 57). A média do diâmetro mínimo do canal foi de 3,05 1,24mm (1 a 8). Avaliações clínicas e ecocardiográficas foram realizadas seriadamente.

Resultados - Implantes adequados ocorreram em 143 $(95,3 \%)$ pacientes. A prevalência de shunt residual imediato foi de $52,1 \%$, decrescendo para $15,9 \%$ no seguimento. Esta taxa caiu para 5,2\% após a realização de procedimentos adicionais. Embolização de coils ocorreu em 12 procedimentos e de umbrella em 1. Um paciente apresentou hemólise e outro estenose discreta da artéria pulmonar esquerda. Não houve mortalidade.

Conclusão - Esta modalidade terapêutica é segurae eficaz, proporcionando bons resultados a longo prazo.
\end{abstract}

Palavras-chave: persistência do canal arterial, cardiologia intervencionista, cardiopatias congênitas

\section{Overall Experience with Percutaneous Occlusion of the Patent Ductus Arteriosus}

Purpose - To evaluate the overall experience of our institution with percutaneous occlusion of the patent ductus arteriosus.

Methods - Since December/92, 150 patients underwent 178 procedures (85 - Rashkind technique; 87 Gianturco coils; 6 - Duct Occlud). Median age was 6.5 years (1 to 57). Mean minimum ductal diameter was $3.05 \pm 1.24 \mathrm{~mm}$ (1 to 8). Clinical and echocardiographic evaluations were performed periodically.

Results - Adequate implantation was achieved in 143 (95.3\%) patients. Prevalence of immediate residual shunting was $52.1 \%$, falling to $15.9 \%$ during follow-up. This figure decreased to $5.2 \%$ after new additional procedures. Umbrella and coil embolization occurred in 1 and 12 procedures, respectively. Hemolysis and mild stenosis of left pulmonary artery were observed in one patient each. There was no mortality.

Conclusion - This therapeutic modality is safe and efficacious with good follow-up results.

Key-words: patent ductus arteriosus, interventional cardiology, congenital heart diseases

Arq Bras Cardiol, volume 71 (nº 6), 769-780, 1998

A persistência do canal arterial, excluindo-se prematuros, corresponde a 5-10\% das cardiopatias congênitas ${ }^{1}$. Após o $1^{\circ}$ ano de vida apresenta taxas anuais de $0,6 \%$ de fechamento espontâneo e de $0,5 \%$ de mortalidade ${ }^{2}$. Além da sua importância clínica, o canal arterial adquiriu notável

Instituto Dante Pazzanese de Cardiologia - São Paulo

Correspondência: Valmir F. Fontes - Instituto Dante Pazzanese de Cardiologia Av. Dr. Dante Pazzanese, 500 - 04012-180 - São Paulo, SP

Recebido para publicação em 24/6/98

Aceito em 14/10/98 valor histórico quando se tornou a primeira anomalia congênita a ser corrigida cirurgicamente por Gross e Hubbard, em $1938^{3}$ e a ser tratada através do cateterismo intervencionista por Porstman e col em $1967^{4}$. No final da década de 70, Rashkind introduziu um novo sistema de oclusão percutânea que se difundiu rapidamente pelo mundo, tornando-se um dos procedimentos intervencionistas com maior documentação científica existente dentro da Cardiologia Pediátrica ${ }^{5-9}$.

Os trabalhos pioneiros de Porstmann e Rashkind deixaram bases sólidas culminando com o advento de novas técnicas ${ }^{10-12}$. Destas, a oclusão do canal através do implan- 
te de molas ou coils de Gianturco é a mais simples e a menos onerosa, tendo se popularizado rapidamente ${ }^{13-16}$.

O objetivo deste trabalho é analisar a experiência global da nossa Instituição no fechamento percutâneo do canal arterial, empregando-se as técnicas de Rashkind, das molas ou coils de Gianturco (Cook Inc, USA) e do oclusor ductal (Duct Occlud, PFM, Germany).

\section{Métodos}

Desde dezembro/92, 83 pacientes foram submetidos a 85 tentativas de implante de umbrellas através da técnica de Rashkind (grupo 1 - G1), desde dezembro/94, 75 pacientes foram submetidos a 87 tentativas de implante de coils (grupo 2 - G2) e desde outubro/96, seis pacientes foram submetidos a seis tentativas de implante do oclusor ductal (grupo 3 - G3), perfazendo um total de 150 pacientes com diagnóstico clínico, ecocardiográfico e angiográfico de canal arterial persistente, submetidos a 178 procedimentos percutâneos. Os riscos e benefícios do procedimento foram esclarecidos aos pacientes ou responsáveis e obtido um consentimento por escrito. A idade variou de 11 meses a 57 (média 7,2 $\pm 8,7$; mediana 6,5) anos, sendo significativamente menor nos pacientes do $\mathrm{G} 2(5,4 \pm 4,3$ anos para o $\mathrm{G} 2 \times 8,6 \pm 8,9$ anos para o G1 x 16,6 $\pm 21,6$ anos para o G3 - p<0,05). O peso variou de 8 a 75 (média 24,6 $\pm 15,5$, mediana 20) $\mathrm{kg}$. Eram do sexo feminino $102(68 \%)$ pacientes. Todos os pacientes encontravam-se em classe funcional (CF) I (NYHA) e, em todos, observou-se sopro contínuo em região infraclavicular esquerda. Na radiografia de tórax, $120(80 \%)$ pacientes apresentavam discreto aumento na área cardíaca e em 30 a área cardíaca foi normal. Todos apresentavam trama vascular aumentada. Ao eletrocardiograma (ECG) constatou-se sobrecarga ventricular esquerda em 120 (80\%). O ecocardiograma bidimensional com doppler colorido confirmou o diagnóstico na totalidade dos casos. Dois pacientes eram portadores de estenose pulmonar valvar associada, sendo submetidos a valvoplastia pulmonar com sucesso no mesmo procedimento ${ }^{17}$, quatro eram portadores de comunicações ventriculares mínimas, dois de estenose subaórtica em membrana, um de duas comunicações interatriais fechadas, percutaneamente, no mesmo procedimento, através do implante de duas próteses de Amplatzer ${ }^{18,19}$, três de síndrome de Down, três de síndrome da rubéola congênita e um de síndrome de Allagille com insuficiência renal crônica, sendo submetido, periodicamente, à hemodiálise.

Após punção de artéria e veia femorais, todos os pacientes foram submetidos a cateterismo direito e esquerdo rotineiro. Uma aortografia na aorta descendente em nível do canal em projeção lateral esquerda foi realizada para caracterização angiográfica do canal arterial, segundo a classificação de Krishenko ${ }^{20}$ (fig. 1), e para mensuração de seu menor diâmetro através do método digital com correção da magnificação. O diâmetro mínimo do canal arterial variou de 1,0 a 8,0mm(1,9a8,0mmnoG1, 1,0 a 6,0mmnoG2 e 1,3 a 3,8mm no G3) com média de 3,05 $\pm 1,24 \mathrm{~mm}$ (mediana 2,9mm), sendo significativamente menor no $\mathrm{G} 2$ e no G3 $(3,68 \pm 1,16 \mathrm{~mm}$ no G1 x
2,26 $\pm 0,81 \mathrm{~mm}$ no G2 x 2,50 $\pm 0,97$ no G3; $<<0,01)$. O canal do tipo A foi o mais freqüente $(95 / 150-63,3 \%)$, seguido do C (29/150-19,3\%), doE(12/150-8,0\%), doB(9/150-6,0\%)edo D (5/150-3,3\%), obedecendo a classificação de Krichenko e $\mathrm{col}^{20}$, sendo o tipo A de morfologia cônica com o segmento mais estreito na inserção pulmonar; tipo B curto e em janela; tipo $\mathrm{C}$ tubular sem constrições; tipo D com múltiplas constrições e o tipo E de forma bizarra.

Na técnica de Rashkind, a via venosa anterógrada foi utilizada rotineiramente para o implante ${ }^{5,9,21}$. A umbrella dupla de Rashkind (USCI, Bard, USA) é constituída de dois discos de poliuretano montados sobre uma armação de aço inoxidável, com três hastes em cada disco para a umbrella $\mathrm{n}^{\circ} 12$ (de $12 \mathrm{~mm}$ ) e quatro hastes para a de $\mathrm{n}^{\circ} 17(17 \mathrm{~mm})$. A umbrella 12 foi utilizada para oclusão de canais menores que $4 \mathrm{~mm}$ e a 17 para canais entre 4 e $8 \mathrm{~mm}^{9,21}$. O sistema de liberação é controlado através de um cateter, especialmente manufaturado para o implante. Bainhas de Mullins longas 8 Fr são necessárias para o implante de umbrellas 12 , e bainhas 11 Fr para as umbrellas $17^{21}$. Em nosso material, aumbrella 12 foi utilizada em 42 procedimentos e a $17 \mathrm{em} 43$. Em dois pacientes, as umbrellas foram implantadas para oclusão de shunts residuais após implante prévio de umbrella.

Os coils são espiras metálicas revestidas por cerdas de dácron que têm alta capacidade trombogênica. Estas espiras são encontradas comercialmente em diversas configurações, variando-se o diâmetro interno da haste metálica, o diâmetro externo da alça e o comprimento total da mola quando esticada $^{21}$. Nesta série de pacientes, de acordo com a disponibilidade do dispositivo, a seleção de uma ou mais molas para oclusão do canal procurou obedecer a seguinte regra: era escolhido um dispositivo com diâmetro externo da alça com pelo menos o dobro do diâmetro interno mínimo do canal e com um comprimento suficiente para produzir a formação de pelo menos três alças ${ }^{14-16,21}$. Todos os procedimentos foram realizados através da via arterial retrógrada clássica, de acordo com protocolos técnicos de implante já previamente descritos ${ }^{15,16,21}$. Neste método, o sistema de liberação não é controlado. Variações da técnica padrão também foram empregadas: em um paciente foram implantados dois coils seqüenciais no mesmo procedimento; em três casos implantamos duas molas simultaneamente (através da via arterial retrógrada por punção de duas artérias femorais) ${ }^{22}$; em três pacientes o procedimento foi assistido com cateter balão wedge pressure ${ }^{23,24}$ e em outros dois implantamos duas molas simultâneas com a ajuda do balão ${ }^{22}$. O diâmetro externo das alças dos coils, mais frequientemente utilizados, foram: 3: 3/87 -3,5\%;4:4/87-4,6\%;5:58/87-66,6\%;8:21/87-24,1\%;e 10: $1 /$ $87-1,2 \%$. Em 14 procedimentos foram utilizados coils com comprimentos suficientes para a formação de apenas duas alças. Em sete pacientes, os coils foram empregados para oclusão de shunts residuais, após implante prévio de umbrellas (um no mesmo procedimento). Em seis procedimentos os coils foram empregados para oclusão de shunts residuais, após implante prévio de coils, sendo que em três pacientes foi necessário a dilatação do canal com cateter de angioplastia coronária para possibilitar a passagem do cateter liberador ${ }^{25}$. 


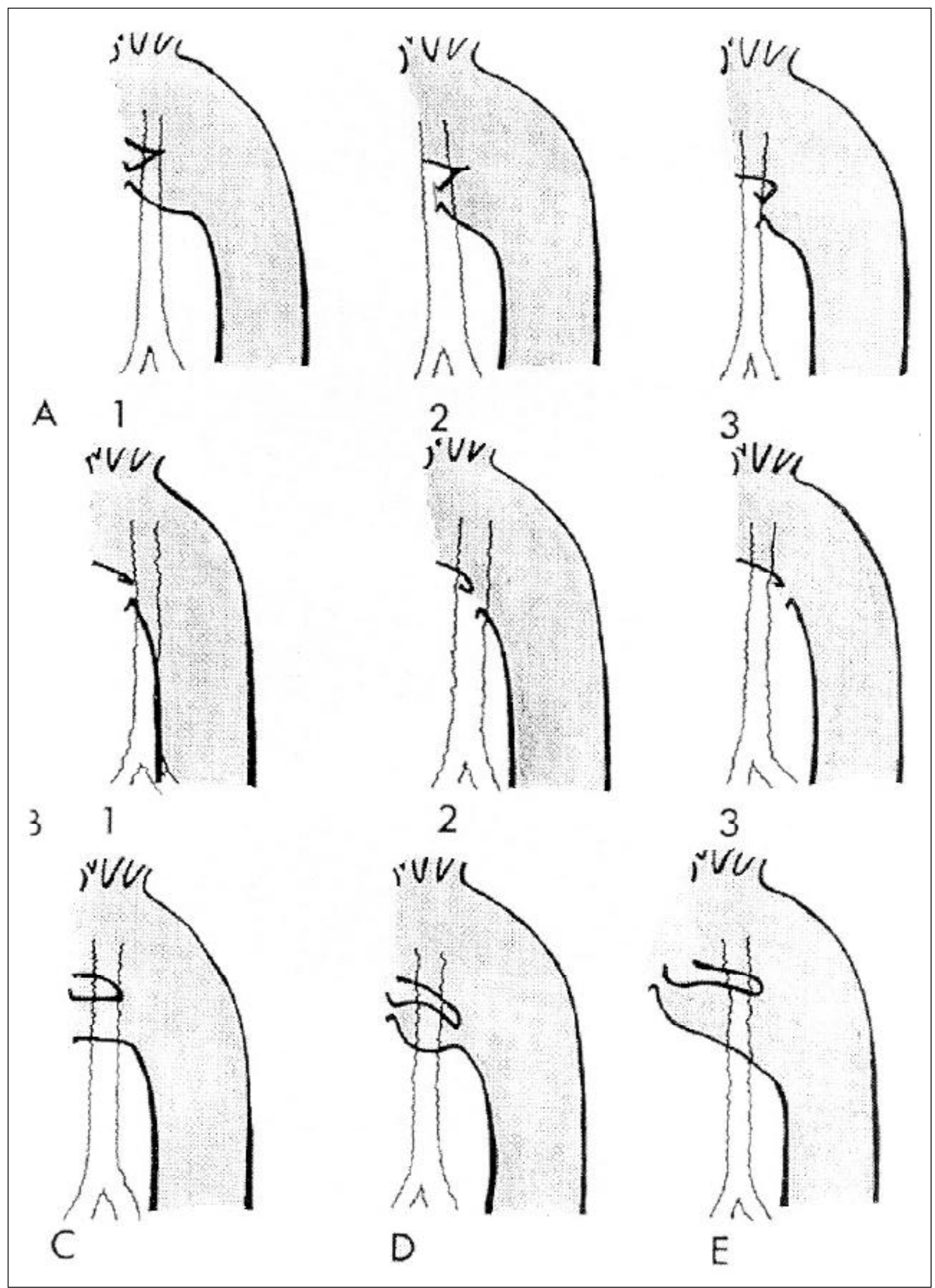

Fig. 1 - Classificação angiográfica dos tipos de canal arterial segundo Krishenko e col (adaptado ${ }^{20}$ ). 
Na técnica de oclusão com o oclusor ductal, o dispositivo constitui-se de uma mola metálica, sem cerdas de dácron, envolvendo a sua superfície que, uma vez implantado, assemelha-se a uma ampulheta, com a região central apresentando um diâmetro discretamente menor que as extremidades. Apresenta inúmeras configurações relativas ao diâmetro externo das alças proximais, mediais e distais e ao seu comprimento total esticado. O implante é realizado através da via venosa anterógrada, com cateteres de baixo perfil, possuindo um sistema de liberação controlado muito seguro ${ }^{26}$. A escolha do dispositivo leva em conta não só o menor diâmero do canal e seu formato anatômico, como também a sua extensão e o diâmetro da ampola ductal em nível da aorta.

Para todas as técnicas, a cefazolina foi empregada profilaticamente e a heparina não foi utilizada rotineiramente antes do procedimento. Uma anestesia geral balanceada (inalatória e venosa) foi utilizada nos pacientes menores de 10 anos, com intubação endotraqueal nos menores de três anos a critério da equipe anestésica. Nas crianças maiores, adolescentes e adultos procuramos realizar o procedimento sob sedação consciente.

Uma angiografia na aorta descendente em projeção lateral esquerda foi realizada cerca de 20min após o implante, a fim de se avaliar a presença de shunt residual imediato ${ }^{9,21}$. De modo geral (excetuando-se dois casos), os portadores de shunt residual imediato não foram submetidos a novo procedimento de oclusão no mesmo tempo, sendo mantidos em acompanhamento. Uma avaliação clínica e ecocardiográfica foi realizada na manhã seguinte do procedimento. A ecodopplercardiografia com mapeamento de fluxo a cores deu ênfase à ausência ou à presença de shunt residual e sua magnitude ${ }^{27,28}$. O shunt residual foi classificado em trivial se só sistólico, discreto se presente também na diástole e restrito à artéria pulmonar esquerda, moderado se acometesse parte do tronco da pulmonar, e grave se atingisse também a valva pulmonar ${ }^{28}$. Medidas das velocidades de fluxo na artéria pulmonar esquerda e na aorta também foram sistematicamente realizadas ${ }^{28}$. Mesmo nos casos de oclusão total imediata ou dentro das primeiras $24 \mathrm{~h}$, ainda foi recomendado, quando necessário, a profilaxia para endarterite infecciosa por cerca de mais três meses, até se completar o processo de endotelização da prótese ${ }^{5}$. Após um mês, os pacientes retornaram para nova avaliação clínica com realização de radiografia de tórax e ECG. Com três e seis meses de acompanhamento, além do exame clínico, foram realizados novos estudos ecocardiográficos e, a partir do $1^{\circ}$ ano após o implante, as consultas clínicas foram realizadas anualmente, juntamente com a realização de radiografia de tórax, ECGe ecocardiograma ${ }^{9}$.

Se após cerca de um ano de seguimento houvesse persistência de shunt residual significativo (definidos pela presença de sopro contínuo ou de shunt maior que trivial ao ecocardiograma), o paciente era encaminhado a novo cateterismo para avaliação da possibilidade de novo implante ${ }^{29}$. As técnicas para os procedimentos adicionais de reoclusão não diferiram substancialmente das utilizadas para a aborda- gem do canal nativo ${ }^{25,30-35}$. Com a possibilidade de dilatação dos shunts residuais diminutos ${ }^{25}$, passamos a encaminhar todos os portadores de shunt residual tardio para uma tentativa de oclusão total após o $1^{\circ}$ ano de seguimento.

Os valores são expressos em média \pm o desvio padrão. $\mathrm{O}$ teste $\mathrm{T}$ de Student e o $\mathrm{x}^{2}$ foram empregados dependendo da indicação. A regressão logística foi aplicada para avaliação de preditores. Um valor de $\mathrm{p} \leq 0,05$ foi considerado como estatisticamente significativo.

\section{Resultados}

Técnica de Rashkind - De 85 tentativas, o dispositivo foi adequadamente implantado em 83 procedimentos (97,6\% de sucesso) (fig. 2). No $1^{\circ}$ paciente da casuística, houve embolização da umbrella para a artéria pulmonar esquerda. Em um outro paciente com canal de diâmetro mínimo de 3,6mm e do tipo C, uma umbrella 12 não ancorou devidamente no canal e o procedimento foi abortado, sem intercorrências. Os dois procedimentos realizados para oclusão de shunts residuais, após implante prévio de umbrella, foram bem sucedidos, com interrupção do fluxo ainda no laboratório de hemodinâmica (fig. 3).
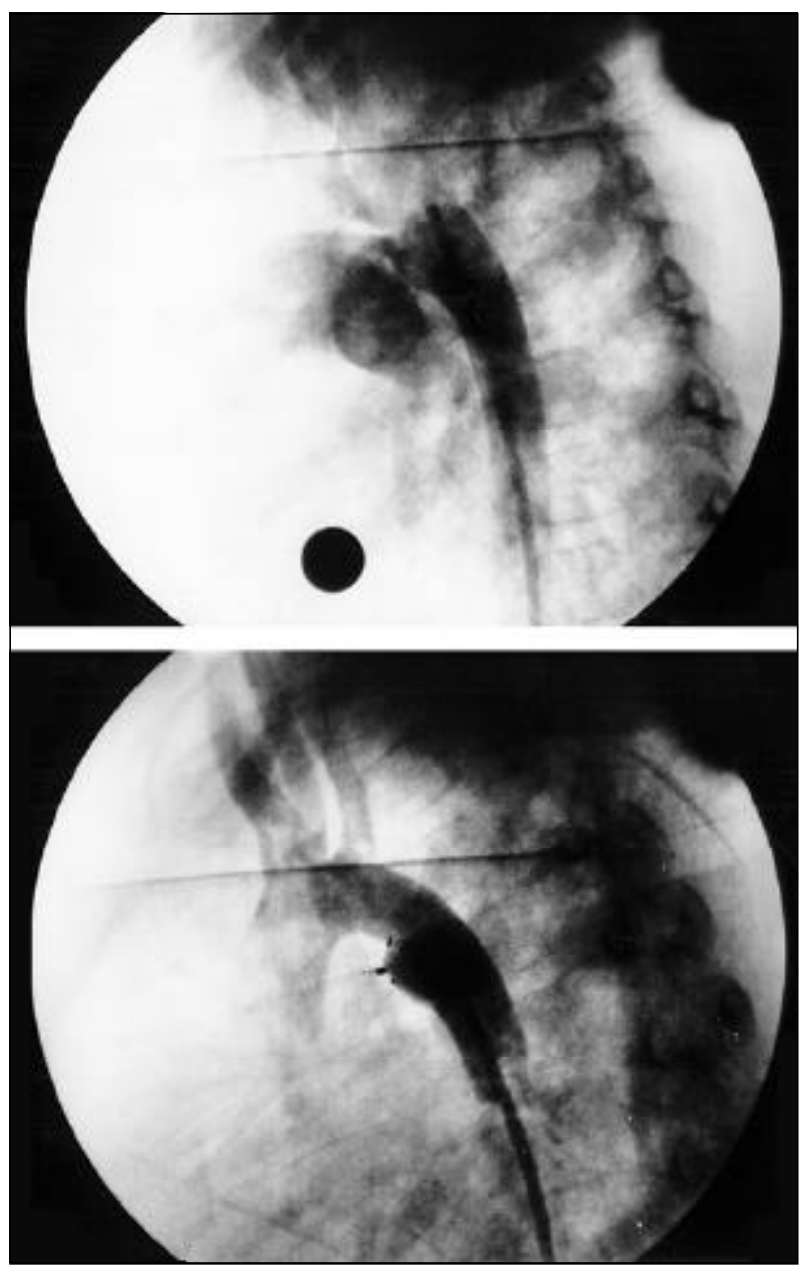

Fig. 2 - Canal arterial de 3,4mm de diâmetro interno mínimo, do tipo A, ocluído com uma umbrella 12 (USCI, Bard, USA), sem shunt residual. 


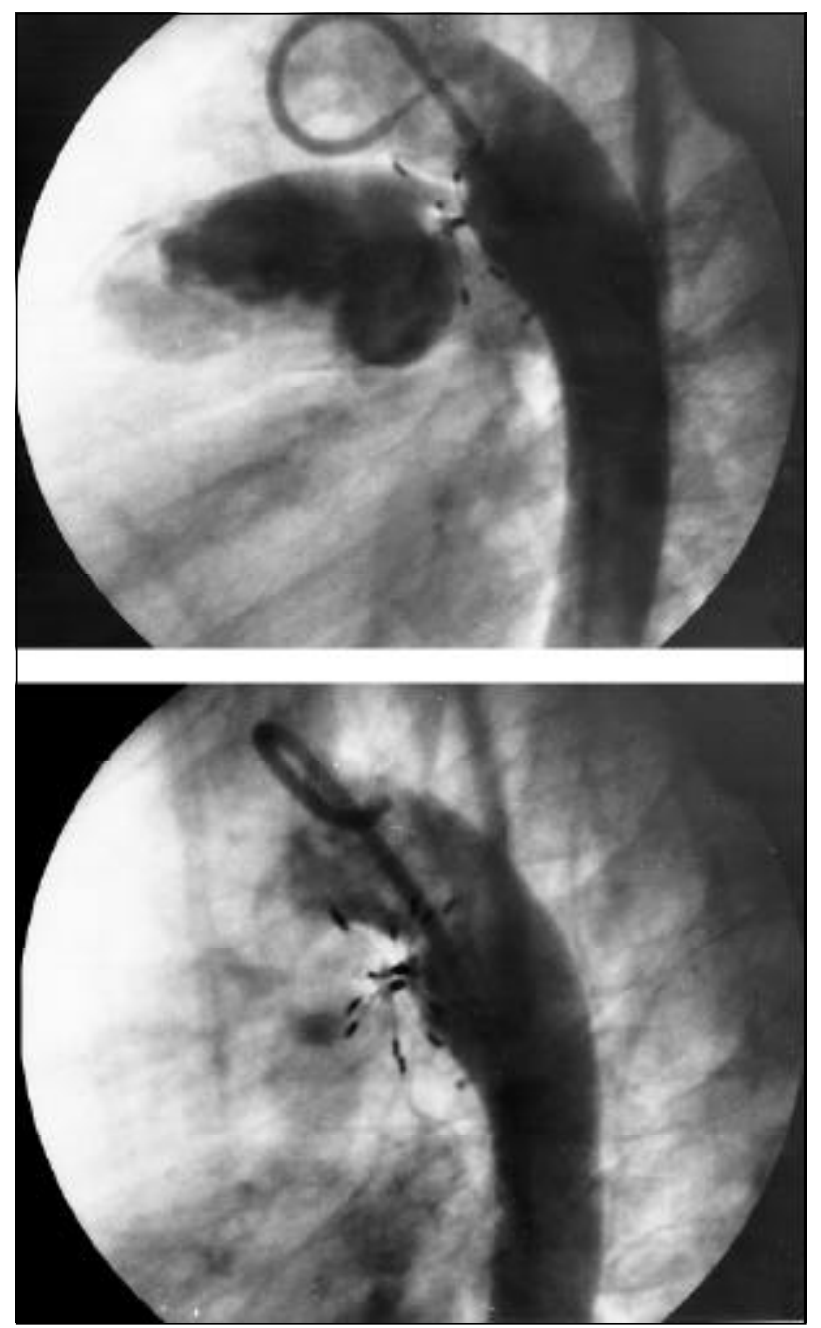

Figura 3 - Shunt residual tardio após implante de umbrella 17, ocluído com um novo implante de umbrella 17 com sucesso e interrupção imediata do fluxo através do canal.

Técnica de coils - De 87 tentativas, as molas foram adequadamente implantadas em 72 procedimentos $(82,7 \%$ de sucesso) (fig. 4). Em três $(3,4 \%)$ pacientes, o posicionamento das molas dentro do canal não foi considerado satisfatório e, portanto, foram imediatamente resgatadas através de cateteres laço. Desses três pacientes, em um foi implantado umbrella 12 no mesmo procedimento com sucesso; um foi submetido a novo implante de coil com sucesso em um procedimento posterior e o $3^{\circ}$ foi encaminhado para cirurgia devido a hemólise secundária a implante de coil sobre umbrella prévia. Em 12 (13,8\%) pacientes houve embolização para artérias pulmonares. $\mathrm{O}$ diâmetro interno mínimo do canal arterial foi significativamente maior nos casos de insucesso: $2,93 \pm 1,18 \mathrm{~mm} \times 2,06 \pm 0,56 \mathrm{~mm}$ ( $\mathrm{p}<0,03)$. A utilização de um coil relativamente curto (35-5-8; suficiente para a formação de apenas duas alças) também esteve relacionado com insucesso (coil curto: nove insucessos em 15 $(60 \%)$ vezes em que foram utilizados x coils longos: seis insucessos em $61(9,8 \%)$ vezes em que foram utilizados $\mathrm{p}<0,001)$. Além disto, dois casos de embolização ocorreram em canais do tipo C (tubular) e um outro em um canal do tipo
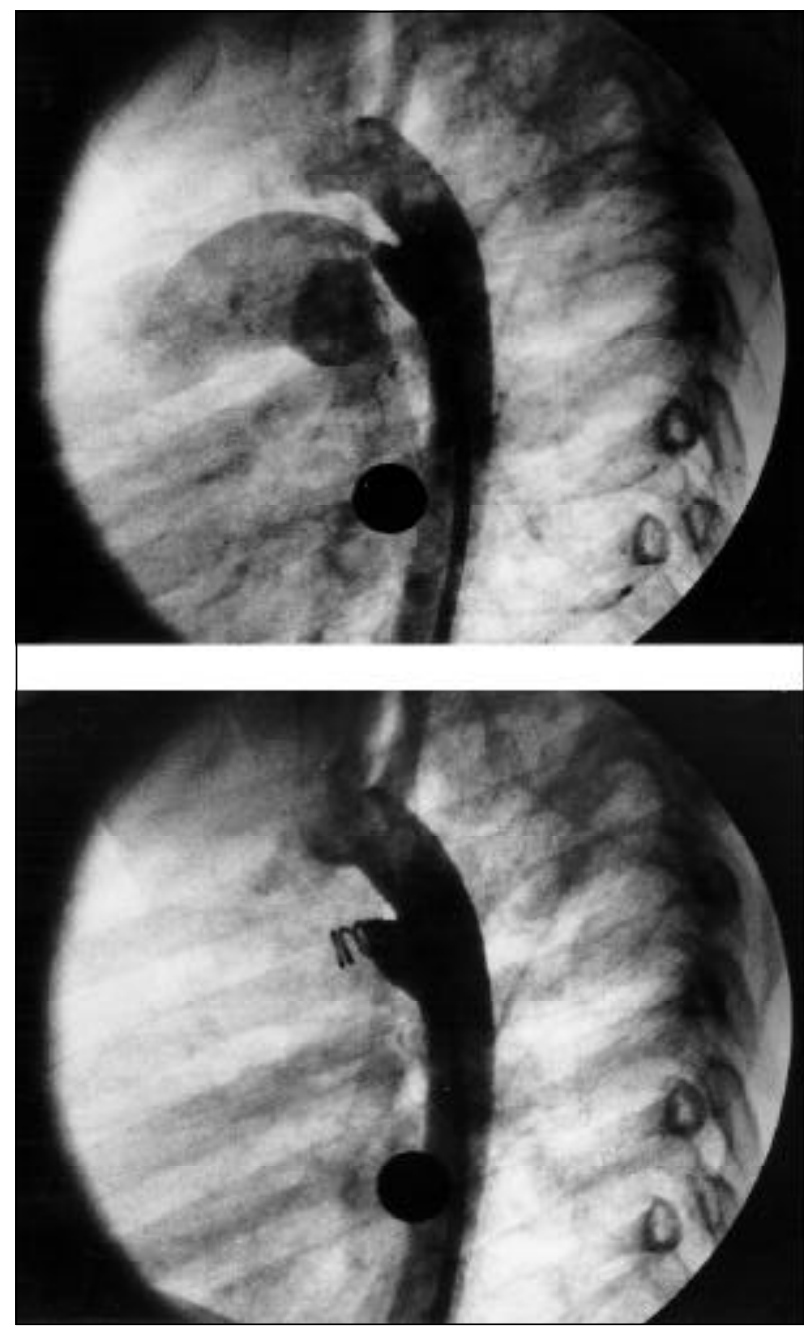

Figura 4 - Canal arterial do tipo A, de 2,3mm de diâmetro, ocluído com coil 38-5-8 (Cook, USA), sem shunt residual imediato.

B (em janela). Dos 15 episódios de insucesso, em 10 encontramos um ou mais fator de risco predisponente para tal (diâmetro maior que $2,5 \mathrm{~mm}$, canal tubular ou em janela ou utilização de um coil relativamente curto). Controlando diversas variáveis (idade, peso, sexo, diâmetro e tipo do canal e o uso do coil de duas alças) através da regressão logística, apenas o diâmetro do canal apareceu como preditor de insucesso no implante $(\mathrm{p}<0,02)$. Dos 12 pacientes em que os coils foram utilizados para oclusão de shunts residuais tardios após implante prévio de umbrella ou de coil, um evoluiu com hemólise e nos outros 11 os procedimentos foram bem sucedidos, com interrupção imediata do fluxo em 8 e tardia em 1 (fig. 5 e 6). Dois pacientes necessitaram de mais um procedimento adicional para oclusão total (totalizando três procedimentos percutâneos cada).

Técnica do oclusor ductal - Das seis tentativas de implante, os dispositivos foram adequadamente implantados em quatro (66,6\%) ocasiões (fig. 7). Nas duas ocasiões em que houve insucesso, as configurações do dispositivo não estavam adequadas para o tipo de canal abordado. As molas foram retiradas facilmente antes de sua liberação final e 


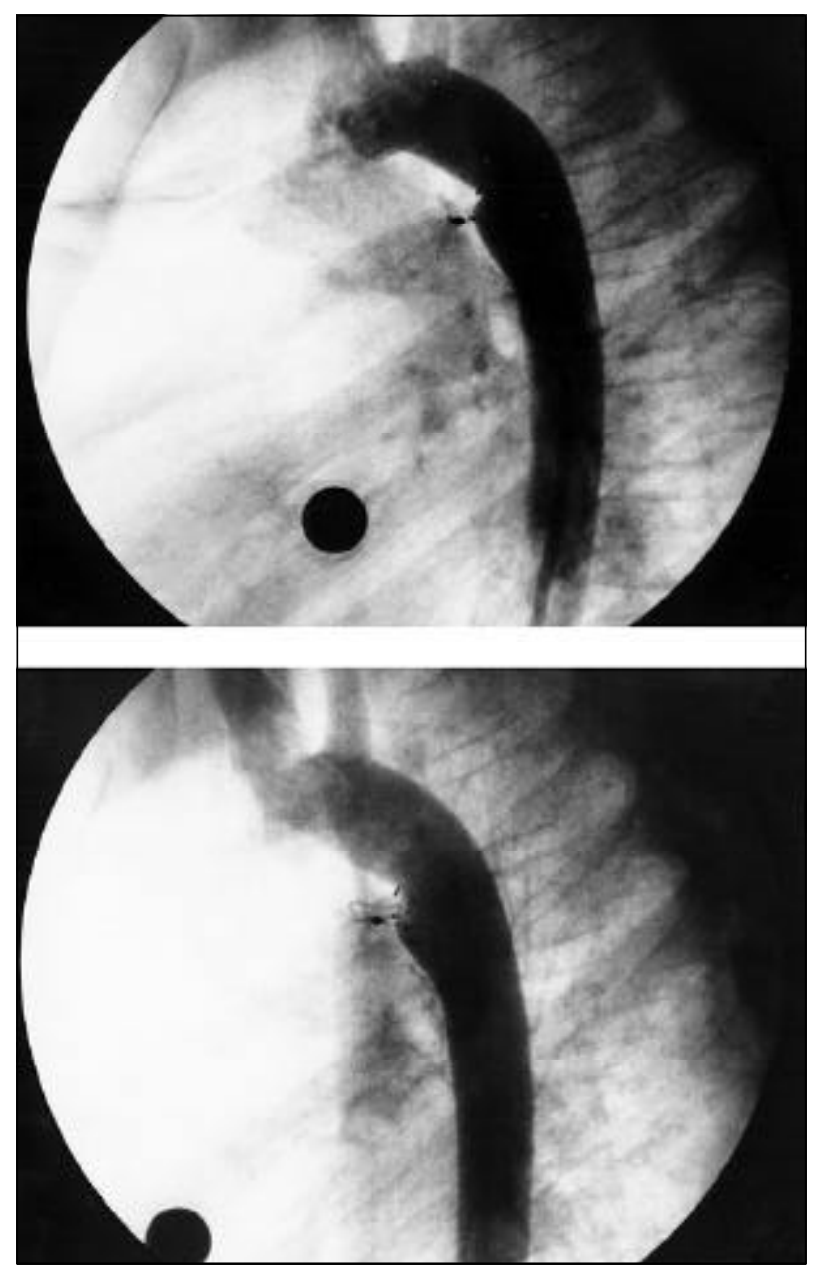

Fig. 5 - Shunt residual tardio após implante de umbrella 17, ocluído com implante adicional de coil (35-5-5) por sobre a umbrella .

os procedimentos foram abortados sem complicações. Estes dois pacientes foram submetidos a implante de umbrellas em outras ocasiões com sucesso.

Complicações - No $1^{\circ}$ paciente da casuística de umbrellas houve embolização do dispositivo (umbrella $\mathrm{n}^{\circ} 17$ ) para a artéria pulmonar esquerda, sendo necessária sua retirada cirúrgica. $\mathrm{O}$ canal do tipo tubular $(\mathrm{C})$ medindo $8 \mathrm{~mm}$ foi seccionado e suturado ${ }^{9}$. No grupo de coils, a taxa de embolização de 13,8\% aparentemente esteve relacionada a uma má seleção de pacientes. Todos os 12 pacientes que sofreram episódios de embolização foram submetidos a angiografia pulmonar seletiva imediata, e em nenhum constatouse interrupções de fluxo em ramos de maior calibre da artéria pulmonar. Em seis pacientes os coils foram resgatados ainda no laboratório de hemodinâmica. Desses seis pacientes, três foram submetidos a implante de umbrella, dois sofreram novos implantes de coils (um ainda no mesmo procedimento) e em um perdeu-se o seguimento. Quatro pacientes permaneceram com um coil na circulação pulmonar, um permaneceu com dois coils e outro com três coils. Desses seis pacientes, quatro foram encaminhados para tratamento ci-

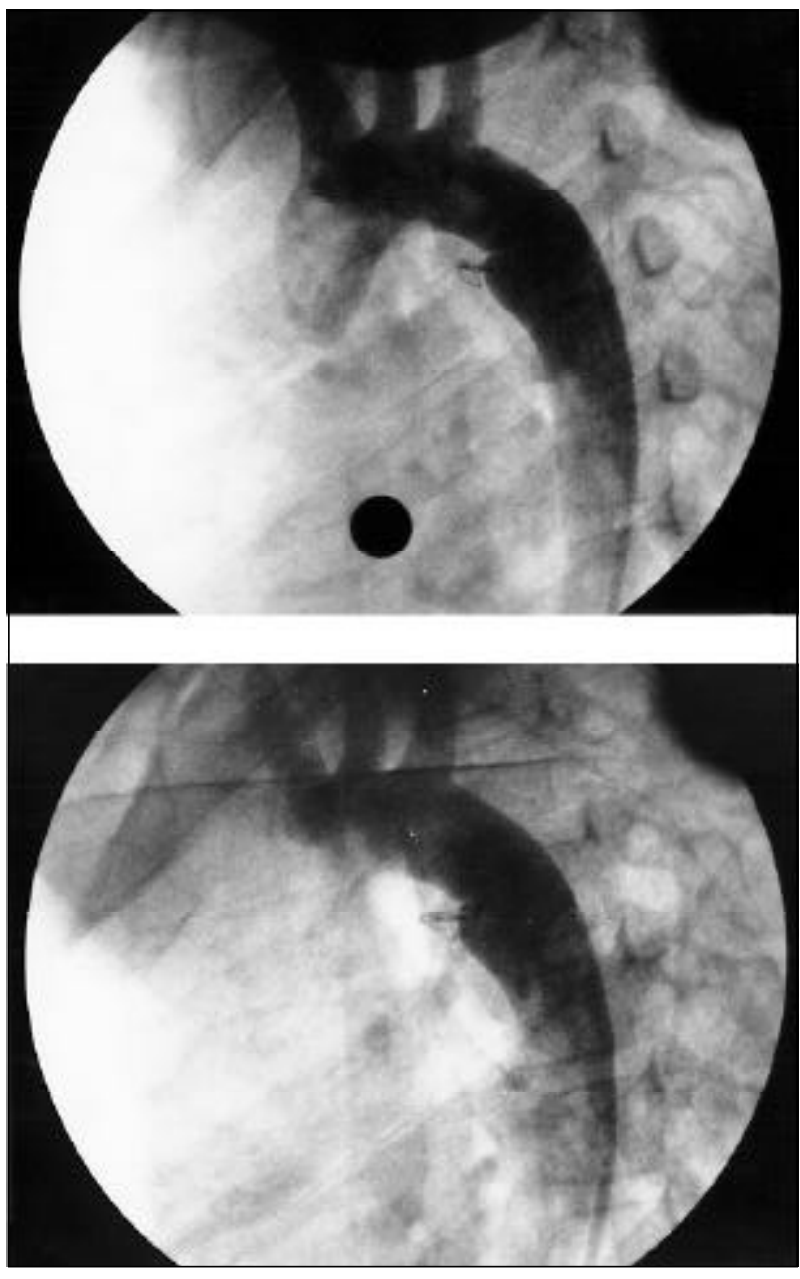

Fig. 6 - Shunt residual tardio mínimo (jato de alta velocidade na porção superior do dispositivo) após implante de coil 35-5-5, ocluído com um novo implante de coil 35-5-5, com sucesso. Neste caso houve a necessidade de dilatação do orifício de shunt com cateter balão de angioplastia coronária para permitir a progressão do cateter liberador.

rúrgico do canal, sendo que em um, após breve período de circulação extracorpórea, resgatou-se um dos dois coils embolizados, em outro a tentativa de resgate não teve sucesso e nos outros dois optou-se apenas pela secção e sutura do canal. Os dois pacientes restantes submeteram-se a implante de umbrella com sucesso. Desses seis pacientes que permaneceram com coils na circulação pulmonar, quatro foram submetidos a mapeamento pulmonar com radioiótopos duas semanas, três, seis e 12 meses após a embolização: em dois a perfusão pulmonar foi considerada normal e, emum, foi notadoum infarto pulmonar laminar mínimo emum segmento anterior do lobo inferior direito. No paciente em que três coils não puderam ser resgatados (localizados em regiões diferentes do pulmão), o mapeamento revelou uma área de infarto discreto a moderado em lobo inferior do pulmão esquerdo relativo ao local de um doscoils (35-5-8). Nas áreas relativas ao posicionamento dos outros dois coils, a perfusão foi considerada normal.

Cinco $(3,4 \%)$ pacientes do G2 evoluíram com perda de pulso distal após o procedimento, necessitando de estreptoquinase na sala recuperação pós-cateterismo para reaber- 

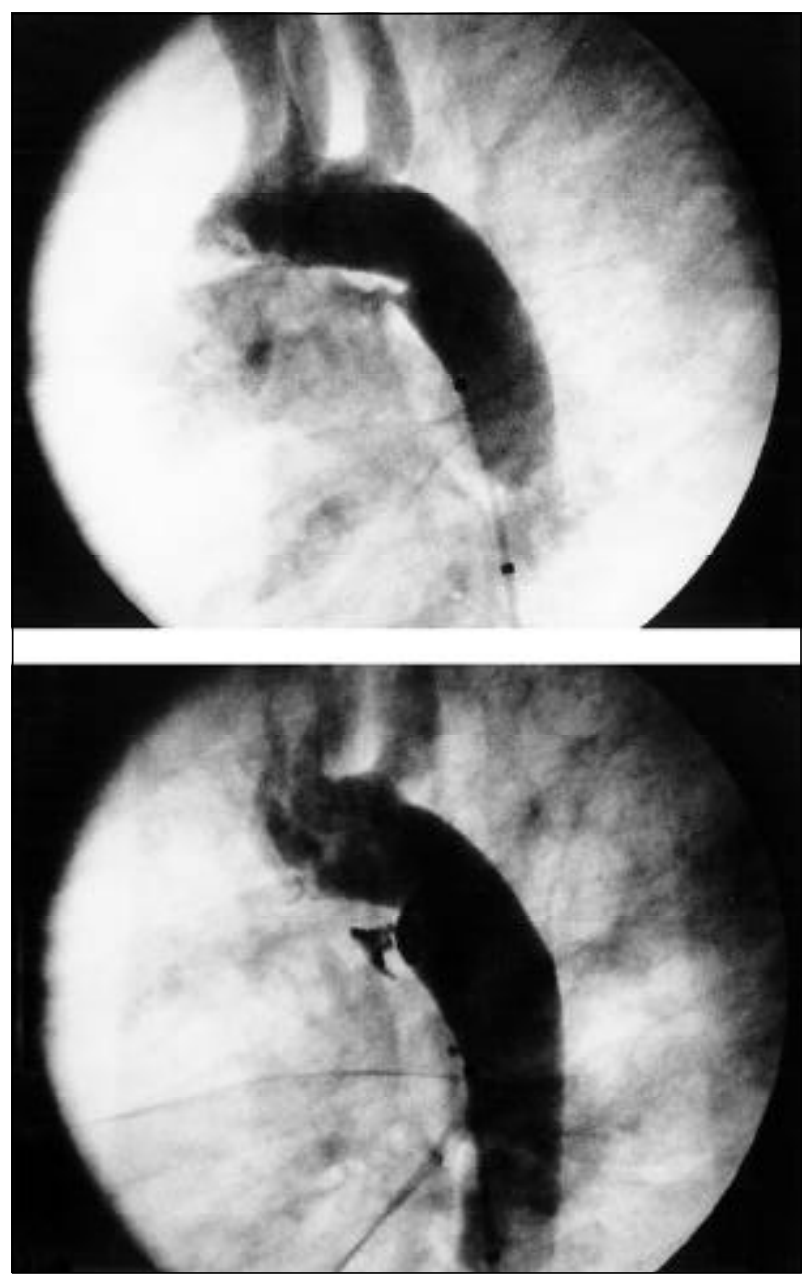

Fig. 7 - Canal arterial de 1,8mm, do tipo A, ocluído com oclusor ductal (Duct Occlud, PFM, Germany), com sucesso, sem shunt residual imediato.

tura da artéria, com sucesso imediato em três. Um outro paciente apresentou retorno espontâneo da amplitude do pulso no seguimento. Em um paciente, um coil que foi resgatado após embolização ficou preso na veia femoral, sendo necessária sua retirada cirúrgica e reparo da veia ainda no laboratório de cateterismo.

Sete $(4,8 \%)$ pacientes necessitaram de transfusão após o procedimento. Um $(0,7 \%)$ paciente evoluiu com sinais clínicos e laboratoriais sugestivos de hemólise intravascular detectados um mês após implante de coil (35-5-10) sobre umbrella prévia. Como o quadro persistiu mesmo após terapêutica transfusional, o paciente foi encaminhado para uma tentativa de implante de um novo coil (35-5-8). Devido a posicionamento inadequado, este $2^{\circ} \mathrm{coil}$ foi resgatado e o paciente, encaminhado a cirurgia, onde ambos os dispositivos foram retirados sem a necessidade de circulação extracorpórea, o canal foi seccionado e suturado e a hemólise controlada ${ }^{36}$.

Não tivemos mortalidade e não observamos nenhum caso sugestivo de endarterite no seguimento imediato e tardio. Foi concedida alta hospitalar na tarde do mesmo dia ou na manhã seguinte do procedimento a $136(90,6 \%)$ pacientes.
Shunt residual imediato - Excetuando os pacientes que não tiveram sucesso no implante e que foram encaminhados à cirurgia $(n=5)$ ou que perderam o seguimento $(n=2)$ $\mathrm{e}$, considerando apenas os procedimentos realizados para a oclusão do canal nativo (sem a existência prévia de dispositivos) e com próteses adequadamente implantadas, a prevalência de shunt residual imediato $20 \mathrm{~min}$ após o implante foi de $61,7 \%$ (50/81) para o G1, 40,4\% (23/57) para o G2, $25,0 \%$ (1/4) para o G3 e de 52,1\% no total. As taxas de shunt residual imediato e tardio relativas aos diferentes subgrupos encontram-se nos gráficos 1 e 2 . As variáveis peso, idade, sexo, diâmetro interno e tipo anatômico do canal, e dimensões do dispositivo foram submetidas a análise uni e multivariável através da regressão logística em cada grupo, não se identificando preditores de shunt residual imediato.

Seguimento, shunt residual tardio e procedimentos de reoclusão - G1: em um paciente do G1 com shunt residual imediato, foi implantado um coil no mesmo procedimento com sucesso e com abolição imediata do shunt. Quatro casos com shunt residual imediato perderam o seguimento clínico. Dos 76 pacientes restantes, 32 apresentaram resolu-

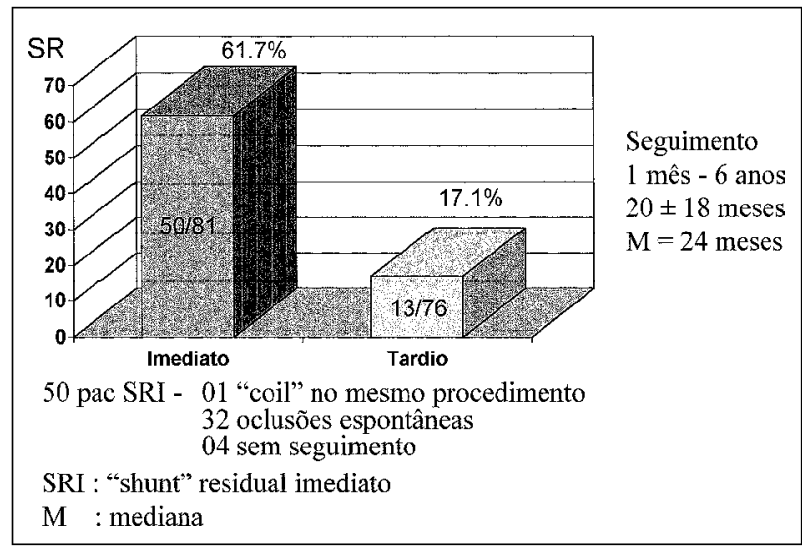

Gráfico 1 - Fechamento espontâneo do shunt residual imediato no grupo 1. Seguimento: 1 mês -6 anos; $20 \pm 18$ meses; M- 24 meses; 50 pacientes SRI- 1 coil no mesmo procedimento; 32 oclusões espontâneas; 4 sem seguimento; SRI- shunt residual imediato; M- mediana.

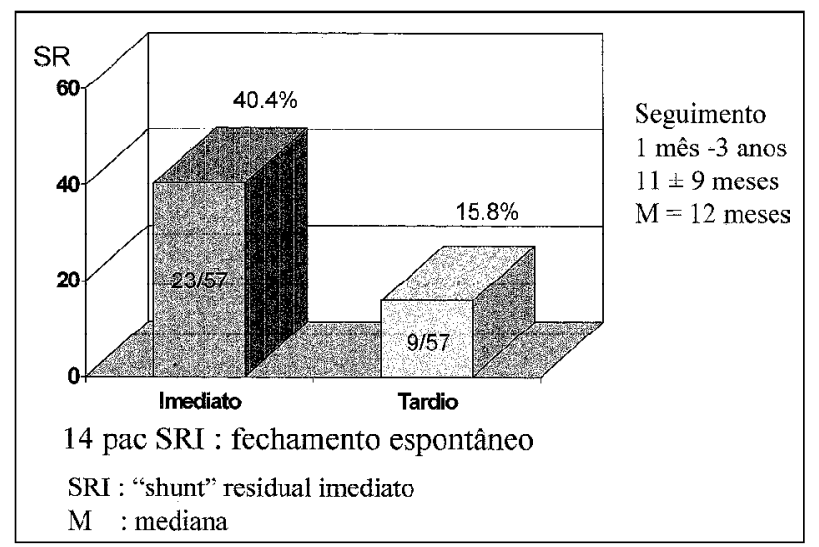

Gráfico 2 - Fechamento espontâneo do shunt residual imediato no grupo 2. Seguimento: 1 mês - 3 anos; $11 \pm 9$ meses; M- 12 meses; 14 pacientes SRI- fechamento espontâneo; SRI- shunt residual imediato; M- mediana. 


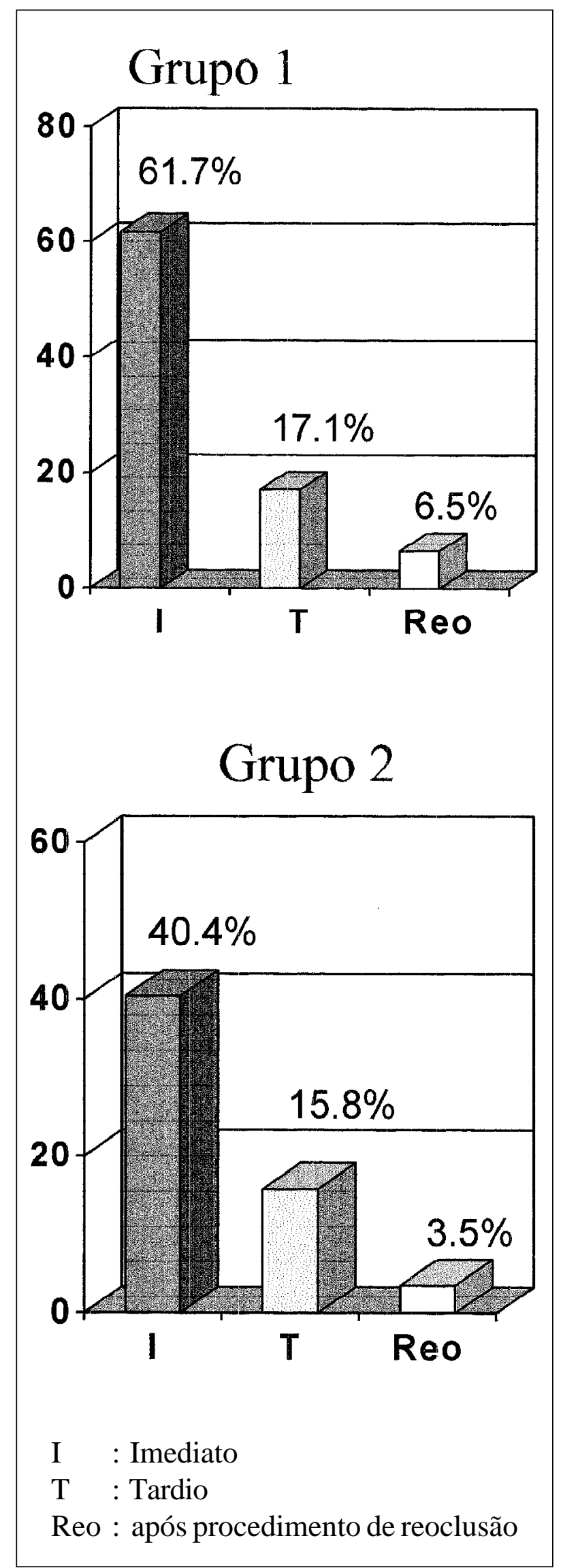

Gráfico 3-Shunt residual tardio após procedimento de reoclusão. I- imediato; Ttardio; Reo- após procedimento de reoclusão. ção espontânea do shunt residual durante o acompanhamento, confirmada pela ecocardiografia com mapeamento de fluxo a cores. Em dois pacientes documentou-se a oclusão espontânea do shunt residual após o $1^{\circ}$ ano de seguimento, em outro, entre seis meses e dois anos e, no restante dos pacientes, este fenômeno ocorreu dentro do $1^{\circ}$ ano de acompanhamento. Portanto, $13(17,1 \%)$ de 76 pacientes apresentavam shunt residual na última consulta (tempo de seguimento um mês a seis anos; média de $20 \pm 18$ meses; mediana 24 meses) (gráfico 1). Nove desses 13 pacientes foram submetidos a tentativas de reoclusão tardia dos shunts residuais. Em um paciente, o procedimento não teve sucesso porque o orifício de shunt era tão pequeno que impedia até a passagem de um fio guia steerable para coronária. Em dois casos, as umbrellas foram utilizadas com abolição imediata do shunt. Em seis pacientes os coils foram utilizados com interrupção imediata do shunt em quatro. Um evoluiu com hemólise e outro necessitou de mais um novo implante de coil (totalizando três procedimentos). Portanto, computando os resultados dos procedimentos adicionais de reoclusão, a taxa de shunt residual tardio no G1 decresceu para $6,5 \%(5 / 76)$, sendo que quatro pacientes ainda apresentam um seguimento inferior a três meses (gráfico 3 ).

G2 - Neste grupo, em um paciente com shunt imediato, foi implantado mais um coil de forma seqüencial, no mesmo procedimento, com abolição imediata do shunt. Quatorze pacientes apresentaram resolução espontânea do shunt residual dentro do $1^{\circ}$ ano de seguimento. Nenhum paciente foi perdido no acompanhamento. Portanto, dos 57 pacientes, $9(15,8 \%)$ apresentavam shunt residual no último seguimento detectados através do ecocardiograma (tempo de seguimento: um mês a três anos; média de $11 \pm 9$ meses; mediana de 12 meses) (gráfico 2). Desses nove pacientes, oito foram submetidos a procedimentos adicionais de implante de coils na tentativa de ocluir completamente o canal. Em dois pacientes o procedimento de reoclusão não teve sucesso devido ao mínimo orifício de shunt. De forma curiosa, em um destes pacientes houve fechamento espontâneo do shunt um ano e meio após a tentativa de reoclusão. Nos outros seis pacientes, os procedimentos tiveram sucesso, com interrupção imediata do shunt em quatro e tardia em um. Um paciente necessitou de mais um novo implante de coil para oclusão completa do canal (totalizando três procedimentos). O outro paciente com shunt residual tardio deverá retornar para consulta de rotina em breve (de dois anos). Portanto, computando os resultados dos procedimentos adicionais de reoclusão, a taxa de shunt residual tardio no G2 decresceu para 3,5\% (2/57) (gráfico 3).

G3 - Todos os quatro pacientes foram acompanhados e um apresentou resolução espontânea do shunt dentro do $1^{\circ}$ ano de seguimento, caracterizando uma taxa de shunt residual tardio de $0 \%$.

As variáveis peso, idade, sexo, diâmetro interno, tipo anatômico do canal, e tamanho do dispositivo foram submetidas a análise uni e multivariável através de regressão logística, não se identificando preditores de shunt residual tardio. As taxas de oclusão imediata e tardia, incluindo os 
procedimentos de reoclusão, relativas aos diferentes subgrupos se encontram nos gráficos 1 e 2 . Para finalizar, computando os resultados finais relativos aos três grupos, dos 137 pacientes com dispositivos adequadamente implantados e efetivamente seguidos, houve oclusão total do canal com um ou mais procedimentos em 130, perfazendo uma taxa final de oclusão de 94,9\%. Não se observaram fenômenos de recanalização tardia do canal.

Seguimento e alterações de fluxo na artéria pulmonare na aorta - Um paciente de 17 anos, submetido a implante de umbrella 17, evoluiu apresentando de forma persistente uma velocidade, estimada através do Doppler pulsado, de cerca de $3,0 \mathrm{~m} / \mathrm{s}$ (gradiente sistólico estimado em $36 \mathrm{mmHg}$ ) na artéria pulmonar esquerda. Em outro paciente de 18 meses $(9 \mathrm{~kg})$, submetido a implante de coil 35-8-5, detectou-se uma velocidade de $2,5 \mathrm{~m} / \mathrm{s}$ na aorta descendente em nível do canal no retorno de três meses após o implante (gradiente estimado de $25 \mathrm{mmHg}$ ).

Dos 150 pacientes submetidos as três técnicas descritas para fechamento percutâneo do canal arterial, o objetivo final (oclusão total) foi obtido em $130(86,7 \%)$. Sete $(4,7 \%)$ pacientes permaneceram comshunt residual no seguimento, apesar do pouco tempo de acompanhamento em quatro. Quatro $(2,7 \%)$ pacientes com shunt residual imediato não foram acompanhados, existindo ainda a possibilidade de fechamento espontâneo tardio. Seis $(4,0 \%)$ pacientes foram encaminhados para tratamento cirúrgico (um devido a hemólise), sendo que em quatro pacientes em que houve embolização de coils, ainda havia a possibilidade de oclusão com umbrellas, não disponíveis no momento da intercorrência. Os dois $(1,3 \%)$ pacientes que tiveram insucesso inicial no implante seriam candidatos a novos implantes se não tivessem perdido o acompanhamento.

\section{Discussão}

O tratamento do canal arterial está indicado em qualquer idade quando acarreta repercussão hemodinâmica de difícil controle clínico, ou é realizado de forma eletiva com o objetivo de prevenir a instalação de endarterite infeccio$\mathrm{sa}^{1,12,29}$. Portanto, o canal arterial detectado clinicamente, através da presença de sopro contínuo em região infraclavicular esquerda e confirmado por estudos ecocardiográficos, tem indicação formal de tratamento, seja ele cirúrgico ou percutâneo ${ }^{12,29}$. Canais diagnosticados acidentalmente através da ecocardiografia, sem achados auscultatórios, são definidos como "canais silenciosos" ${ }^{37}$. Estima-se que estejam presentes em cerca de $0,5 \%$ de pacientes assintomáticos, sem suspeita de cardiopatia e são submetidos a exame ecocardiográfico por motivos não cardíacos. O tratamento deste subgrupo de pacientes permanece controverso ${ }^{12,25,29}$. A incidência de endarterite infecciosa em um "canal silencioso" é excepcional ${ }^{38}$. A maioria dos autores ${ }^{12,39,40}$ não recomenda o tratamento rotineiro destes pacientes. No entanto parece-nos prudente recomendar a profilaxia para endarterite infecciosa sempre que necessário ${ }^{29}$. As contraindicações de tratamento do canal arterial correspondem a doença vascular pulmonar oclusiva, já instalada, e a presença de cardiopatia congênita cianogênica canal dependente ${ }^{12}$.

Por possuir um sistema de liberação controlado, o índice de sucesso no implante de umbrellas é alto $(97,6 \%)$ e a ocorrência de embolização incomum ${ }^{5-9}$. Analisando-se, retrospectivamente, nossos casos de insucesso, o $1^{\circ}$ paciente deveria ser submetido a tratamento cirúrgico devido ao grande diâmetro do canal. No $2^{\circ}$ paciente, como o canal possuía diâmetro próximo a $4 \mathrm{~mm}$ e uma morfologia tubular, uma umbrella 17 e não a 12 é que deveria ter sido utilizada. Nosso índice de shunt residual imediato é semelhante à casuística mundial e não teve relação com nenhum fator preditivo ${ }^{5-9}$.

Desde o relato inicial de Cambier e col ${ }^{13}$, vários autores descreveram suas experiências com o fechamento percutâneo do canal arterial através do implante de molas de Gianturco com resultados variados ${ }^{14-16}$. Em um estudo multicêntrico, relatando os resultados imediatos de 535 procedimentos, o índice de sucesso no implante chegou a $95 \%{ }^{41}$. Oclusão completa nas primeiras $24 \mathrm{~h}$ foi obtida em $75 \%$ dos pacientes e oclusão parcial em $20 \%$. Nossos índices encontram-se um pouco aquém desses devido à seleção inadequada de pacientes e de dispositivos em alguns casos (enganos inerentes a curva de aprendizado inicial). Um outro motivo é que, possivelmente, em alguns casos de insucesso, as medidas do canal não foram estimadas apropriadamente devido à natureza elástica do canal arterial ${ }^{29}$. Esta característica dinâmica de sua estrutura é um fenômeno comum na prática intervencionista diária e que pode levar a escolhas inapropriadas de quaisquer dispositivos para seu fechamento. Neste mesmo estudo multicêntrico, foram identificados como fatores preditivos para uma maior incidência de shunt residual imediato, um diâmetro maior tanto do canal arterial quanto da espira. Estes fatores não foram identificados em nossa série, talvez devido ao tamanho da amostragem. Alguns autores adotam uma postura de tolerância zero a presença de shunt residual imediato, ou seja: implantam-se quantos coils forem necessários até a oclusão total imediata do canal ${ }^{14,42}$.

Por não possuir um sistema de liberação controlada, a ocorrência de embolização é comum, com taxas variando entre 5 a $20 \%$, sendo mais freqüente durante a curva de aprendizado ${ }^{12,22,29,31,41}$. Um diâmetro maior do canal e canais arteriais do tipo $\mathrm{B}$ e C são considerados como preditores de insucesso no implante ${ }^{41}$. Na nossa experiência, estes fatores também foram identificados. Nossos casos de insucesso estiveram relacionados a um diâmetro maior do canal arterial, dois episódios de embolização ocorridos em canais do tipo C (tubular) e outro em um do tipo B (em janela), de anatomias desfavoráveis para o necessário ancoramento do coil, devido à ausência de constrições ${ }^{43}$. Um aspecto técnico que também teve influência negativa nos resultados desta experiência foi a utilização de molas relativamente curtas, permitindo a formação de apenas duas alças. Molas com comprimentos suficientes para a formação de no mínimo três alças (de preferência até de quatro a cinco alças) devem ser utilizadas de rotina ${ }^{12}$. Em grande parte dos casos, os coils embolizados podem ser resgatados ainda na própria 
sala de cateterismo ${ }^{14-16}$. Os que, por algum motivo, não são resgatados, geralmente acabam se localizando em pequenos ramos da árvore pulmonar, não trazendo maiores conseqüências clínicas ${ }^{43}$. Os resultados da angiografia pulmonar realizada nos 12 pacientes, logo após a embolização, e os resultados do mapeamento pulmonar com radioisótopos, realizados no seguimento em quatro pacientes, sustentam, em parte, esta observação. Em nenhum caso houve interrupções de fluxo para ramos maiores da artéria pulmonar e dos pacientes submetidos a mapeamento, em dois notouse perfusão pulmonar normal, em um havia mínima área de infarto e em outro com três coils embolizados para locais distintos da árvore pulmonar, observamos uma área de infarto relativa apenas a um coil. Este fenômeno talvez possa ser explicado pela orientação espacial que as molas assumem dentro dos vasos após sua liberação. Conforme estejam posicionadas, em relação ao fluxo sangüíneo, muitas vezes nem chegam a obstruir o vaso ${ }^{44}$.

A fim de reduzir os episódios de embolização, alguns autores utilizam cateteres com um laço em sua extremidade para auxiliar no implante ${ }^{45}$. Com isto, através de via venosa anterógrada, o laço segura e manipula o coil no lado pulmonar, antes de sua liberação final. Outros autores preconizam a via venosa anterógrada rotineiramente para o procedimento, abordando inclusive canais acima de 2,5-3,0mm, através do implante seqüencial de múltiplos coils comótimos resultados ${ }^{14,42}$. Com o intuito de diminuir a freqüência de embolização, temos experimentado variações na técnica clássica de implante, como por exemplo a liberação simultânea de dois coils e o implante assistido com um cateter balão tipo wedge pressure ${ }^{22,24}$. Uma casuística maior com estas variações é necessária para avaliar seu real impacto na minimização desta complicação. Os sistemas de liberação controlada de coils (Jackson detachable coil - Cook ou Duct Occlud PFM), apesar de mais caros, praticamente extinguiram os episódios de embolização ${ }^{26,46}$. Na nossa limitada experiência com o oclusor ductal (Duct Occlud) a segurança proporcionada pelo método pôde ser constatada. No entanto, tivemos impressão que a escolha da prótese ideal não é tão fácil como para as técnicas de umbrella ou coil de Gianturco e que o seu posicionamento adequado dentro do canal requer uma maior manipulação dos cateteres e do dispositivo comparado as outras técnicas, podendo estender o tempo de procedimento.

Outras complicações mais raras, como hemólise, obstrução da artéria pulmonar esquerda ou pseudo coacrtação de aorta descritas em outras séries ${ }^{6-8,12,47-49}$, também foram observadas em nossa casuística. $\mathrm{O}$ caso que evoluiu com hemólise após implante de coil por sobre uma umbrella, provavelmente foi relacionada a seleção inapropriada do dispositivo, permitindo uma protusão excessiva da parte distal da mola em direção à aorta ${ }^{36}$. Em apenas um paciente detectamos uma real estenose na artéria pulmonar esquerda. Este paciente é um candidato a implante de stent para alívio desta lesão. Aumentos discretos da velocidade de fluxo na artéria pulmonar esquerda podem ser observados nas primeiras semanas após o implante com tendência retornar ao normal durante o seguimento. Este declínio provavelmente decorre do processo de epitelização progressiva dos dispositivos ${ }^{28,49}$. Um paciente evoluiu com um aumento significativo da velocidade de fluxo na aorta e, apesar de se encontrar ainda no $3^{\circ}$ mês de seguimento, com possibilidade de regressão desta velocidade, pelo mesmo motivo já citado, este fenômeno pode estar relacionado com a escolha de um coil mais longo (com cinco alças) para uma criança relativamente pequena $(9 \mathrm{~kg})^{42}$.

A prevalência global de shunt residual imediato nos nossos pacientes foi de 52,1\% (74/142). Durante o seguimento, esta taxa declinou de forma espontânea e significativa para 15,9\% (22/138). Uma endotelização progressiva da superfície dos dispositivos e eventuais mudanças em suas geometrias no interior do canal são hipóteses para explicar tal fenômeno ${ }^{6}$. A literatura refere um maior diâmetro do canal como preditor de shunt residual tardio ${ }^{6,7,50}$, o que não foi observado em nossa casuística .

Como ficou constatado em nossa série, shunts residuais tardios podem ser tratados com sucesso através de novos implantes ${ }^{30-35}$. Abordagem que permitiu uma redução significativa na taxa de shunt residual final para 5,2\% (130/ 137). Do ponto de vista técnico, às vezes é necessário dilatar o canal com um cateter de angioplastia coronária a fim de possibilitar a passagem do cateter liberador ${ }^{25}$, evidenciado em três casos. O momento ideal para se indicar o procedimento de reoclusão é assunto discutível na literatura, sendo definido arbitrariamente. Mesmo reconhecendo que o fechamento espontâneo pode levar mais de um ano ${ }^{6}$ (observado em três pacientes do G1), temos nos posicionado da seguinte forma: já que na maioria absoluta dos casos, o fenômeno de fechamento espontâneo dos shunts residuais ocorre durante $\mathrm{o} 1^{\mathrm{o}}$ ano de seguimento se, aproximadamente, após um ano de evolução ainda não houver oclusão total do canal, encaminhamos o paciente para um $2^{\circ}$ procedimento, independente dos achados clínicos ou ecocardiográfi$\cos ^{25}$. Os pacientes que permanecem sem sopros e com shunts triviais ao ecocardiograma também podem ser considerados como portadores de "canal silencioso" 40 . Os três pacientes em que os procedimentos de reoclusão não tiveram sucesso devido ao mínimo orifício de shunt, poderiam ser avaliados também sob esse prisma. Interessante foi notar como um desses pacientes apresentou fenômeno de oclusão espontânea tardia do canal. E aqui cabe um comentário, traçando um paralelo em relação ao que já foi dito sobre esse subgrupo de pacientes: como no momento não há conhecimento suficiente para definir se a presença de um material estranho intravascular pode ou não alterar a história natural, aparentemente benigna, desta entidade, recomenda-se que esses pacientes com shunt residual mínimo e "silencioso" continuem a receber profilaxia antimicrobiana para endarterite infecciosa, quando necessária ${ }^{29}$. Apesar de estarem fora de risco para desenvolvimento de doença vascular pulmonar, esses pacientes constituem-se em uma limitação dos métodos de fechamento percutâneo ${ }^{6}$. A possibilidade de encaminhá-los para tratamento cirúrgico deve ser individualizada. 
As vantagens deste tipo de abordagem são óbvias. $\mathrm{O}$ fechamento percutâneo do canal arterial proporciona um curto período de internação $(24 \mathrm{~h})$, podendo até ser realizado em caráter ambulatorial ${ }^{51}$; elimina a ocorrência de dor e desconforto geral relacionados à toracotomia; não implica em uma cicatriz cirúrgica anti-estética e por fim, minimiza consideravelmente o forte impacto negativo que qualquer procedimento terapêutico invasivo causa sobre o binômio psicoafetivo e social do paciente ${ }^{9}$. A técnica de coils emprega um material (dispositivo, introdutor, cateteres e fio guia) de uso rotineiro no laboratório de hemodinâmica, possibilitando uma redução considerável no custo do procedimento ${ }^{21}$. Além disto, a técnica é simples e de fácil aprendizado. Os introdutores utilizados são de baixo perfil ( $3 \mathrm{~F}$ a $6 \mathrm{~F})$, o que permite sua utilização em pequenos lactentes. Pequenos canais arteriais podem ser ocluídos sem dificuldades técnicas, não sendo necessárias adaptações técnicas ${ }^{52}$. O método de Rashkind, apesar de oferecer maior segurança ao procedimento, tem custos um pouco mais elevados, às vezes comparáveis aos do tratamento cirúrgico ${ }^{53}$. Ao nosso ver, acreditamos que em países em desenvolvimento, com poucos centros especializados no tratamento de cardiopatias congênitas, o fechamento percutâneo também tem o mérito de possibilitar um alívio na sobrecarga operatória desses centros ${ }^{7}$, reservando a abordagem cirúrgica para o tratamento de cardiopatias congênitas mais complexas $^{9}$.

Qualquer técnica designada para o fechamento percutâneo do canal arterial leva em conta a idade e o peso do paciente, o diâmetro mínimo do canal arterial e seus aspectos anatômicos baseados na classificação de Krishenko ${ }^{20}$. As técnicas aqui apresentadas apresentam algumas limitações. No método de Rashkind, por exemplo, um peso inferior a 6kg impede a utilização dos introdutores necessários para o implante ( 8 Fr ou $11 \mathrm{Fr})^{5-9}$. Pacientes nesta faixa de peso também são de risco para desenvolvimento de estenose da artéria pulmonar esquerda ${ }^{54}$. Canais maiores que $8 \mathrm{~mm}$ são considerados de grande risco para embolização e, portanto, devem ser tratados cirurgicamente ${ }^{29}$.

Em geral são candidatos a oclusão com coils de Gianturco através da técnica convencional (implante de único coil pela via arterial retrógrada), portadores de canais de até 2,5-3,0mm de diâmetro interno mínimo, de forma cônica (tipo A), com múltiplas constrições (tipo D) ou de configuração aberrante (tipo E) (ou seja, aqueles que têm uma ampola aórtica bem definida e/ou constrições no trajeto para ancorar bem o coil) ${ }^{29,43}$. Acima desse diâmetro e em canais do tipo B e $\mathrm{C}$, o risco de insucesso é grande. Os coils também constituem-se em ótima alternativa para a oclusão de shunts residuais tardios após implante prévio de outras próteses ${ }^{25,30-35}$. Nestas situações, o risco de embolização fica minimizado pela presença do outro dispositivo no canal ${ }^{25,33}$.

Pelos motivos já expostos, neonatos e lactentes sintomáticos, portadores de canais calibrosos (felizmente infreqüentes) não são candidatos ao fechamento percutâneo através das técnicas aqui descritas. O advento de novas próteses, como por exemplo, a Amplatzer (AGA Medi- cal Corporation, USA) permitiu a extensão do procedimento para pequenos lactentes por requererem bainhas de baixo perfil, por possuírem dimensões compactas e se adaptarem a qualquer tipo anatômico de canal. Este dispositivo proporciona taxas de oclusão de $100 \%$ em um seguimento de três meses (Dr. Ziyad Hijazi - comunicação pessoal), estando ainda em fase inicial de estudos clínicos (quase 100 casos).

Mesmo tendo um caráter pouco invasivo, o fechamento percutâneo do canal arterial não é $100 \%$ eficaz, deixando um pequeno grupo com shunts residuais mínimos, sem repercussão clínica e com risco ainda desconhecido para endarterite (provavelmente muito baixo) ${ }^{6}$.É importante notar que a experiência aqui relatada possui uma natureza dinâmica devido a curva de aprendizado e a possibilidade dos shunts residuais ainda poderem apresentar fechamento espontâneo ou serem ocluídos através de novos procedimentos. Assim sendo, consideramos que o fechamento percutâneo do canal arterial através das técnicas aqui descritas apresenta resultados finais extremamente satisfatórios, podendo ser encarado como uma alternativa terapêutica inicial segura e eficaz para pacientes selecionados com canal arterial. Muito provavelmente, o desenvolvimento tecnológico permitirá a abordagem percutânea de qualquer tipo de canal em praticamente todas as faixas etárias (o neonato continuará sendo um desafio), aumentando a simplicidade técnica, segurança e eficácia do método com otimização da relação custo/benefício.

\section{Agradecimentos}

À Srta Angela Paes pela análise estatística.

\section{Adendo}

Após o término da configuração deste manuscrito, mais cinco procedimentos de implante de coil foram realizados para oclusão do canal arterial. Um dos pacientes com shunt residual tardio do grupo de coil retornou após dois anos de acompanhamento, apresentando shunt trivial ao ecocardiograma, sendo submetido a novo implante de coil com oclusão total imediata. Um outro paciente com implante prévio de coil apresentou fenômeno de recanalização após dois anos de seguimento e foi submetido a novo implante de coil, com abolição imediata doshunt, que era trivial ao ecocardiograma. Em ambos os casos, devido à natureza diminuta do orifício de shunt, foi necessária a dilatação prévia do canal com cateter balão para angioplastia coronária para permitir a passagem do cateter liberador. Mais três pacientes com canais nativos entre 2,0 e $2,8 \mathrm{~mm}$ foram submetidos a implantes de coil (um com implante simultâneo de dois coils) com oclusão imediata em dois, confirmados pela ecocardiografia. Dados que corroboram com os resultados já apresentados, reduzindo ainda um pouco mais a taxa de shunt residual tardio no grupo 2 para $3,3 \%$ (com um paciente com tempo de seguimento inferior a um mês). 


\section{Referências}

1. Brook MM, Heymam MA - Patent ductus arteriosus. In: Emmanouilides GC, Riemenschneider TA, Allen HD, Gutgesell HP. eds) - Heart Disease in Infants, Children and Adolescents. Including the Fetus and Young Adult. $5^{\text {th }}$ ed. Baltimore: William \& Wilkins, 1995; 746-64.

2. Campbell M-Natural history of persitent ductus arteriosus. Br Heart J 1968; 30: 4-13.

3. Gross RE, Hubbard JP - Surgical ligation of a patent ductus arteriosus. Report of the first successful case. JAMA 1939; 112: 729-33.

4. Portsmann W, Wierny L, Warnke H, Gerstbrger G, Romanuik PA - Catheter closure of patent ductus arteirosus. 62 cases treated without thoracotomy. Radiol Clin North Am 1971; 9: 201-13.

5. Rashkind WJ, Mullins CE, Hellenbrand WE, Tait MA - Non surgical closure of patent ductus arteriosus: clinical application of the Rashkind PDA occluder system. Circulation 1987; 75: 583-592.

6. Hosking MCK, Benson LN, Musewe N, Dick ID, Freedom RM - Transcatheter occlusion of persistently patent ductus arteriosus. Forty month follow-up and prevalence of residual shunting. Circulation 1991; 84: 2313-17.

7. Ali Khan MA, Yousef AL, Mullins CE, Sawyer W - Experience with 205 procedures of transcatheter closure of ductus arteriosus in 182 patients, with special reference to residual shunts and long-term follow-up. J Thorac Cardiovasc Surg 1992; 104: 1721-27.

8. Tynan M - Report of the European Registry. Transcatheter occlusion of persistent arterial duct. Lancet 1992; 340: 1062-6.

9. Fontes VF, Pedra CAC, Esteves CA et al - Cierre percutáneo del conducto arterioso con la técnica de Rashkind. Experiencia inicial del Instituto Dante Pazzanese de Cardiologia, São Paulo-Brasil. Arch Inst Cardiol Mex 1996; 66: 129-137.

10. Verin VE, Saveliev SV, Kolody SM, Prokubovsky VI - Results of transcatheter closure of the patent ductus arteriosus with the Botallo occluder. J Am Coll Cardiol 1993; 22: 1509-14

11. Rao PS, Sideris PB, Haddad J et al - Transcatheter occlusion of patent ductus arteriosus with ajustable buttoned device. Initial Clinical Experience. Circulation 1993; 88: 1119-1126.

12. Rao PS, Sideris EB - Transcatheter occlusion of patent ductus arteriosus: State of the Art. J Invasive Cardiol 1996; 8: 278-88.

13. Cambier PA, Kirby WC, Worthan DC, Moore JW - Percutaneous closure of the small $(<2,5 \mathrm{~mm})$ patent ductus arteriosus using coil embolization. Am J Cardiol 1992; 69: 815-6.

14. Hijazi ZM, Geggel RL - Results of anterograde transcatheter closure of patent ductus arteriosus using single or multiple Gianturco coils. Am J Cardiol 1994; 74: 925-9.

15. Lloyd TR, Fedderly R, Mendelsohn AM, Samdhu SK, Beeckman RH III Transcatheter occlusion of patent ductus arteriosus with Gianturco coils. Circulation 1993; 88: 1412-20.

16. Moore JW, George L, Kirkpatrick SE - Percutaneous closure of the small patent ductus arteriosus using occluding spring coils. J Am Coll Cardiol 1994; 23: 759-65.

17. Pedra CAC, Pedra SRFF, Esteves CA, Braga SLN, Fontes VF - Tratamento simultâneo da estenose pulmonar valvar e da persistência do canal arterial através do cateterismo intervencionista. Arq Bras Cardiol 1997; 68: 357-62.

18. Pedra CAC, Pedra SRFF, Esteves CA, Assef JE, Fontes VF, Hijazi ZM - Multiple atrial septal defects and patent ductus arteriosus: successful outcome using two Amplatzer septal occluders and Gianturco coils. Cath Cardiovasc Diagn 1998. Submitted to editorial evaluation.

19. Fontes VF, PedaCAC, PedraSRFF-Experiência inicial no fechamento percutâneo da CIA com a prótese de Amplatzer. Arq Bras Cardiol 1998 (no prelo).

20. Krishenko A, Benson LN, Burrows P, Moes CAF, MC Laughlin P, Freedom RM - Angiographic classification of the isolated, persistently patent ductus arteriosus and implications for percutaneous catheter occlusion. Am J Cardiol 1989; 63: 877-80

21. Fontes VF, Esteves CA, Braga SLN, Santana MVT, Silva MAP, Pedra CAC Oclusão do canal arterial: "umbrellas" e "coils" - quando indicar e quais os resultados. Rev Soc Cardiol Est SP 1995; 5: 494-502.

22. Esteves CA, Pedra CAC, Braga SLN, Fontes VF - Percutaneous occlusion of the arterial duct by simultaneous retrograde release of 2 Gianturco coils. J Inv Cardiol. Submitted to editorial evaluation.

23. Dalvi B, Goyal V, Narula D, Kulkarni H, Ramakantan R - New technique using temporary balloon occlusion for transcatheter closure of patent ductus arteriosus with Gianturco coils. Cathet Cardiovasc Diagn 1997; 41: 62-70.

24. Esteves CA, Pedra CAC, Braga SLN, Fontes VF - Implante assistido de mola de Gianturco no canal arterial através da ajuda de cateter balonado. Rev Bras Cardiol Inv 1997; 5: 6-11.

25. Pedra CAC, Esteves CA, Pedra SRFF, Braga SLN, Fontes VF- Indicações, técnica, resultados e impacto clínico dos procedimentos para oclusão de shunts residuais após fechamento percutâneo do canal arterial. Arq Ins Cardiol Mex 1998. Submetido a avaliação editorial.
26. Tometzki A,Chan K, Giovani J et al - Total UK multi-centre experience with a novel arterial occlusion device (Duct Occlud PFM). Heart 1996; 76: 520-4.

27. Musewe NN, Benson LN, Smallhorn JF, Freedom RM - Two dimensional echocardiographic and color flow Doppler evaluation of a ductal occlusion with the Rashkind prothesis. Circulation 1989; 80: 1706-10.

28. Pedra SRFF, Pedra CAC, Jesus C - O papel da ecocardiografia no fechamento percutâneo do canal arterial. Rev Bras Eco 1998. Submetido a avaliação editorial.

29. Pedra CAC, Esteves CA, Braga SLN, Kambara A, Fontes VF- Oclusão percutânea do canal arterial. Estado da Arte. Rev Bras Cardiol Inv 1997; 2: 22-35.

30. Huggon LC, Tabatabaei AH, Qureshi AS - Use of a second transcatheter Rashkind arterial duct occluder for persistent flow after implantation of the first device: indications and results. Br Heart J 1993; 69: 544-50.

31. Moor MD, Al Fadley F, Galal O-Closure of residual leak after umbrella occlusion of the patent arterial duct, using Gianturco coils. Int J Cardiol 1996; 56: 5-9.

32. Moore JW, George L, Kirkpatrick SE - Closure of residual patent ductus arteriosus with occluding spring coil after implant of a Rashkind occluder. Am Heart J 1994; 127: 943-45.

33. Hijazi ZM, Geggel RL, Al-Fadley F - Transcatheter closure of residual patent ductus arteriosus shunting after the Rashkind occluder device using single or multiple Gianturco coils. Cathet Cardiovasc Diagn 1995; 36: 255-8.

34. Abbag F, Galal O, Fadley F, Oufi S - Reocclusion of rersidual leaks after transcatheter occlusion of patent ductus arteriosus. Eur J Pediatr 1995; 154: 518-21

35. Moore JW - Repeat use of occluding spring coils to close residual patent ductus arteriosus. Cathet Cardiovasc Diagn 1995; 35: 172-5.

36. Pedra CAC, Pedra SRFF, Fontes VF - Hemolysis associated with coil occlusion of the arterial duct. Cardiol Young, 1998; 8: 63-6.

37. Houston AB, Guanapragasan JP, Lim MH, Doig WB, Coleman EM - Doppler ultrasound and the silent ductus. Br Heart J 1991; 65: 97-9.

38. Balzer DT, Spray TL, MC Mullin D, Cottingham W, Canter CE - Endarteritis associated with a clinically silent patent ductus arteriosus. Am Heart J 1993; 125: 1192-3.

39. LLoyd TR, Beekman RH III - Clinically silent patent ductus arteriosus (Letter). Am Heart J 1994; 127: 1664.

40. Latson LA - Residual shunts after transcatheter closure of patent ductus arteriosus. A major concern or benign "techno-malady"?. Circulation 1991; 84: 2591-3.

41. Lloyd TR, Beekman RH II, Moore JW et al - The PDA coil registry: Report of the first 535 procedures. Circulation 1995; 92: I-380.

42. Hijazi ZM, Geggel RL - Transcatheter closure of patent ductus arteriosus using coils. Am J Cardiol 1997; 79: 1279-80.

43. Pauperio HM, Redington AN, Rigby ML - Closing the patent arterial duct plugs, umbrellas and coils. Cardiol Young 1996; 6: 252-4.

44. Fellows KE, Lock JE - Catheter intervention: septostomy, occlusion techniques and pericardial drainage. In: Lock JE, Keane JF, Fellows KE, ed - Diagnostic and interventional catheterization in congenital heart disease. : Martinus Nijhoff Publishing, 1987: 128.

45. Sommer RJ, Gutierrez A, Lai WW, Parness IA - Use of preformed Nitinol snare to improve transcatheter coil delivery in occlusion od patent ductus arteriosus. Am J Cardiol 1994; 74: 836-9.

46. Tometzki A, Arnold R, Peart I et al - Transcatheter occlusion of the patent ductus arteriosus with Cook detachable coils. Heart 1996; 76: 531-5.

47. Ladusans EJ, Murdach I, Franciosi - Severe haemolysis after percutaneous closure of a ductus arteriosus (arterial duct). Br Heart J 1989; 61: 548-50.

48. Ottenkamp J, Hess J, Talsma MD, Buis-Lien TN - Protusion of the device: a complication of catheter closure of patent ductus arteriosus. Br Heart J 1992; 68: 301-3.

49. Magee AG, Stumper O, Burns JE, Godman M-Medium term follow up of residual shunting and potential complications after transcatheter occlusion of the ductus arteriosus. Br Heart J 1994; 71: 63-9.

50. Shim D, Fedderly RT, Beekman RH III et al -Follow up of coil occlusion of patent ductus arteriosus. J Am Coll Cardiol 1996; 28: 207-11.

51. Wessel DL, Keane JF, Parness I, Lock JE - Outpatient closure of patent ductus arteriosus. Circulation 1988; 77: 1068-71.

52. Benson LN, Dyck JD, Hecht B - Technique for closure of the small patent ductus arteriosus using the Rashkind occluder. Cathet Cardiovasc Diagn 1988; 14: 82-4.

53. Gray DT, Fyler DC, Walker AM, Weinstein MC, Chalmers TC-Clinical outcomes and costs of transcatheter as compared with surgical closure of patent ductus arteriosus. The patent ductus arteriosus closure comparative study group. $\mathrm{N}$ Engl J Med 1993; 329: 1570-2.

54. Nykanen DG, Hayes AM, Benson LN, Freedom RM - Transcatheter patent ductus arteriosus: application in the small child. J Am Coll Cardiol 1994, 23 : 1666-70. 